\title{
An accessible platform for high-throughput COVID-19 molecular diagnostics and genome sequencing using a repurposed $3 D$ printer for RNA extraction
}

Koen Vandelannoote ${ }^{1, \#}$, Andrew H. Buultjens ${ }^{l}$, Lucy Li ${ }^{1}$, Liam K. Sharkey ${ }^{l}$, Marion Herisse ${ }^{l}$, Sacha J. Pidot ${ }^{1}$, Tuyet Hoang ${ }^{2}$, Benjamin P. Howden ${ }^{1,2,3}$, Ian R. Monk ${ }^{1}$, Torsten Seemann ${ }^{1,2}$, Jean Y. H. Lee $e^{1,4}$, Timothy P. Stinear ${ }^{1, \#}$

1. Department of Microbiology and Immunology, Doherty Institute for Infection and Immunity, University of Melbourne, Melbourne, Victoria, Australia.

2. Microbiological Diagnostic Unit Public Health Laboratory, Doherty Institute for Infection and Immunity, University of Melbourne, Melbourne, Victoria, Australia.

3. Department of Infectious Diseases, Austin Health, Heidelberg, Victoria, Australia

4. Department of Infectious Diseases, Monash Health, Clayton, Victoria, Australia.

\# Corresponding authors: koen.vandelannoote@unimelb.edu.au, tstinear@unimelb.edu.au

Number of Pages (including this cover): 6

Number of figures: 0

Number of Tables: 3 
Table S1: Ender $V X-500$ intra- and inter-instrument precision

\begin{tabular}{|c|c|c|c|c|c|c|}
\hline & & & & E-gen & T-qPCR & \\
\hline & & $\mathbf{N}$ & Min Ct & $\operatorname{Max} C t$ & $\operatorname{Avg~Ct}$ & $\operatorname{Ct~CV(\% )}$ \\
\hline & Ender $V X-5003$ & 23 & 29.76 & 30.69 & 30.11 & $0.91 \%$ \\
\hline $\overrightarrow{0}$ & Ender $V X-5004$ & 23 & 29.84 & 30.63 & 30.20 & $0.66 \%$ \\
\hline E & Ender VX-500 5 & 23 & 29.43 & 31.00 & 30.02 & $1.32 \%$ \\
\hline & Ender VX-500 6 & 23 & 29.32 & 30.53 & 29.85 & $1.08 \%$ \\
\hline & Ender VX-5003_A & 8 & 29.76 & 30.08 & 29.92 & $0.32 \%$ \\
\hline & Ender $V X-5003$ B & 8 & 29.79 & 30.26 & 29.98 & $0.49 \%$ \\
\hline & Ender VX-5003_C & 7 & 30.13 & 30.69 & 30.44 & $0.62 \%$ \\
\hline & Ender VX-500 4_A & 8 & 29.84 & 30.23 & 30.00 & $0.47 \%$ \\
\hline & Ender VX-5004_B & 8 & 30.15 & 30.32 & 30.25 & $0.20 \%$ \\
\hline E & Ender VX-5004_C & 7 & 30.16 & 30.63 & 30.37 & $0.54 \%$ \\
\hline 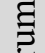 & Ender VX-500 5_A & 8 & 29.50 & 29.99 & 29.82 & $0.51 \%$ \\
\hline & Ender VX-500 5_B & 8 & 29.43 & 30.03 & 29.76 & $0.74 \%$ \\
\hline & Ender VX-500 5_C & 7 & 30.05 & 31.00 & 30.49 & $0.89 \%$ \\
\hline & Ender VX-5006_A & 8 & 29.32 & 29.70 & 29.50 & $0.42 \%$ \\
\hline & Ender VX-500 6_B & 8 & 29.60 & 30.18 & 29.98 & $0.72 \%$ \\
\hline & Ender VX-5006_C & 7 & 29.74 & 30.53 & 30.07 & $0.84 \%$ \\
\hline
\end{tabular}


Table S2: XPRIZE COVID-19 Testing proficiency sample results

\begin{tabular}{|c|c|c|c|c|c|c|}
\hline Material & Concentration & ConcUnits & Volume & Matrix & Ender VX500 E-gene RTqPCR Ct & PE Chemagic Egene RTqPCR Ct \\
\hline Zepto particles & 0 & copies/ul & 400 & $1 \times$ PBS & nd & nd \\
\hline Zepto particles & 0 & copies/ul & 400 & $1 \times$ PBS & nd & nd \\
\hline Zepto particles & 0 & copies/ul & 200 & Nasal & nd & nd \\
\hline Zepto particles & 0 & copies/ul & 200 & Nasal & nd & nd \\
\hline Zepto particles & 0 & copies/ul & 200 & Saliva & nd & nd \\
\hline Zepto particles & 0 & copies/ul & 200 & Saliva & nd & nd \\
\hline Zepto particles & 0.1 & copies/ul & 200 & $1 \times$ PBS & nd & nd \\
\hline Zepto particles & 0.1 & copies/ul & 200 & $1 \times$ PBS & nd & nd \\
\hline Zepto particles & 0.1 & copies/ul & 200 & $1 x$ PBS & nd & nd \\
\hline Zepto particles & 0.1 & copies/ul & 200 & Saliva & nd & nd \\
\hline Zepto particles & 0.1 & copies/ul & 200 & Saliva & nd & 35.19 \\
\hline Zepto particles & 0.1 & copies/ul & 200 & Nasal & nd & nd \\
\hline Zepto particles & 0.1 & copies/ul & 200 & Nasal & nd & 36.04 \\
\hline Zepto particles & 0.5 & copies/ul & 200 & $1 \times$ PBS & nd & nd \\
\hline Zepto particles & 0.5 & copies/ul & 200 & $1 \times$ PBS & 35.51 & nd \\
\hline Zepto particles & 0.5 & copies/ul & 200 & $1 x$ PBS & nd & nd \\
\hline Zepto particles & 0.5 & copies/ul & 200 & Nasal & nd & nd \\
\hline Zepto particles & 0.5 & copies/ul & 200 & Nasal & nd & 36.06 \\
\hline Zepto particles & 0.5 & copies/ul & 200 & Saliva & 35.02 & nd \\
\hline Zepto particles & 0.5 & copies/ul & 200 & Saliva & 34.41 & 34.42 \\
\hline Zepto particles & 1 & copies/ul & 200 & $1 \times$ PBS & 34.74 & 35.71 \\
\hline Zepto particles & 1 & copies/ul & 200 & $1 \times$ PBS & nd & 36.05 \\
\hline Zepto particles & 1 & copies/ul & 200 & $1 x$ PBS & nd & nd \\
\hline Zepto particles & 1 & copies/ul & 200 & Nasal & 34.78 & 36.04 \\
\hline Zepto particles & 1 & copies/ul & 200 & Nasal & nd & 36.23 \\
\hline Zepto particles & 1 & copies/ul & 200 & Saliva & 33.14 & 34.17 \\
\hline Zepto particles & 1 & copies/ul & 200 & Saliva & 34.96 & 33.72 \\
\hline Zepto particles & 2 & copies/ul & 200 & $1 \times$ PBS & 34.84 & 34.21 \\
\hline Zepto particles & 2 & copies/ul & 200 & $1 \times$ PBS & 33.71 & 36.6 \\
\hline Zepto particles & 2 & copies/ul & 200 & $1 x$ PBS & 34.54 & 33.21 \\
\hline Zepto particles & 2 & copies/ul & 200 & Nasal & 33 & 32.34 \\
\hline Zepto particles & 2 & copies/ul & 200 & Nasal & 35.07 & 33.32 \\
\hline Zepto particles & 2 & copies/ul & 200 & Saliva & 33.73 & 33.2 \\
\hline Zepto particles & 2 & copies/ul & 200 & Saliva & 31.74 & 31.42 \\
\hline Zepto particles & 5 & copies/ul & 200 & $1 x$ PBS & 32.47 & 32.39 \\
\hline Zepto particles & 5 & copies/ul & 200 & $1 \times$ PBS & 32.92 & 31.8 \\
\hline Zepto particles & 5 & copies/ul & 200 & $1 x$ PBS & 33.01 & 32.16 \\
\hline Zepto particles & 5 & copies/ul & 200 & Saliva & 33.2 & 30.77 \\
\hline Zepto particles & 5 & copies/ul & 200 & Saliva & 33.1 & 31.21 \\
\hline Zepto particles & 5 & copies/ul & 200 & Nasal & 31.13 & 32.77 \\
\hline Zepto particles & 5 & copies/ul & 200 & Nasal & 32.27 & 31.88 \\
\hline Zepto particles & 10 & copies/ul & 200 & $1 \times$ PBS & 31.88 & 30.51 \\
\hline Zepto particles & 10 & copies/ul & 200 & $1 \times$ PBS & 32.38 & 30.19 \\
\hline Zepto particles & 10 & copies/ul & 200 & $1 x$ PBS & 30.32 & 30.26 \\
\hline Zepto particles & 10 & copies/ul & 200 & Saliva & 30.42 & 29.75 \\
\hline Zepto particles & 10 & copies/ul & 200 & Saliva & 32.35 & 29.89 \\
\hline Zepto particles & 10 & copies/ul & 200 & Nasal & 32.61 & 30.28 \\
\hline Zepto particles & 10 & copies/ul & 200 & Nasal & 32.17 & 31.28 \\
\hline Zepto particles & 25 & copies/ul & 200 & Saliva & 29.98 & 28.99 \\
\hline Zepto particles & 25 & copies/ul & 200 & Saliva & 28.88 & 28.6 \\
\hline Zepto particles & 25 & copies/ul & 200 & Nasal & 30.36 & 29 \\
\hline Zepto particles & 25 & copies/ul & 200 & Nasal & 30.53 & 29.33 \\
\hline Zepto particles & 25 & copies/ul & 200 & $1 \times$ PBS & 28.91 & 28.3 \\
\hline Zepto particles & 25 & copies/ul & 200 & $1 x$ PBS & 28.84 & 28.67 \\
\hline Zepto particles & 25 & copies/ul & 200 & $1 \times$ PBS & 27.79 & 28.58 \\
\hline Zepto particles & 50 & copies/ul & 200 & $1 x$ PBS & 28.99 & 28.14 \\
\hline Zepto particles & 50 & copies/ul & 200 & $1 \times$ PBS & 29.32 & 28.39 \\
\hline Zepto particles & 50 & copies/ul & 200 & $1 x$ PBS & 29.49 & 28.29 \\
\hline Zepto particles & 50 & copies/ul & 200 & Nasal & 28.93 & 28.16 \\
\hline Zepto particles & 50 & copies/ul & 200 & Nasal & 28.43 & 28.31 \\
\hline Zepto particles & 50 & copies/ul & 200 & Saliva & 29.21 & 28.35 \\
\hline Zepto particles & 50 & copies/ul & 200 & Saliva & 28.68 & 27.68 \\
\hline Zepto particles & 100 & copies/ul & 200 & $1 x$ PBS & 27.53 & 27.24 \\
\hline Zepto particles & 100 & copies/ul & 200 & $1 \times$ PBS & 27.08 & 26.45 \\
\hline Zepto particles & 100 & copies/ul & 200 & $1 x$ PBS & 27.76 & 27.46 \\
\hline Zepto particles & 100 & copies/ul & 200 & Saliva & 28.55 & 25.84 \\
\hline Zepto particles & 100 & copies/ul & 200 & Saliva & 30.23 & 26.59 \\
\hline Zepto particles & 100 & copies/ul & 200 & Nasal & 29.83 & 26.86 \\
\hline Zepto particles & 100 & copies/ul & 200 & Nasal & 27.47 & 27.37 \\
\hline
\end{tabular}

Note: nd $=$ not detected 


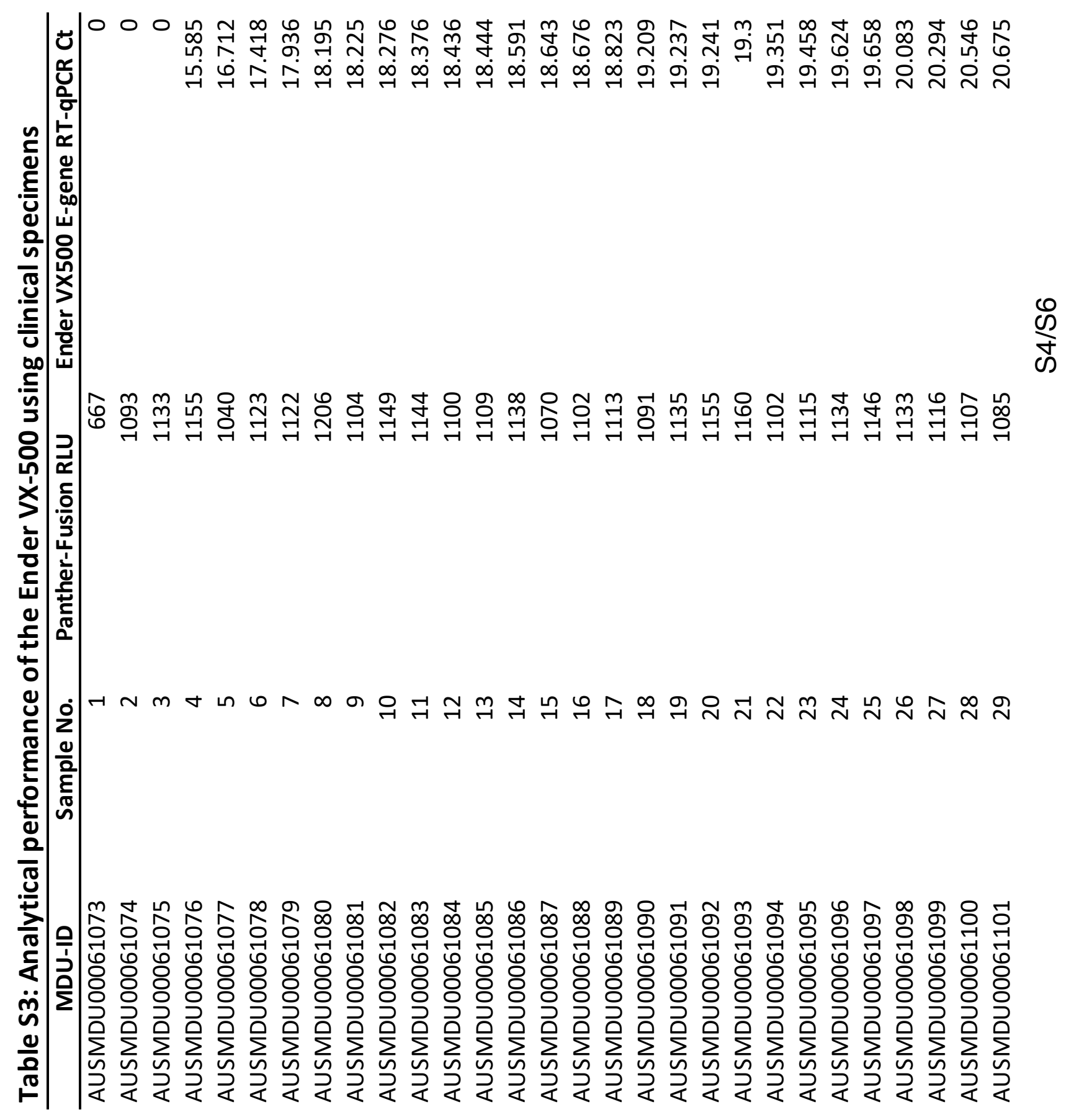




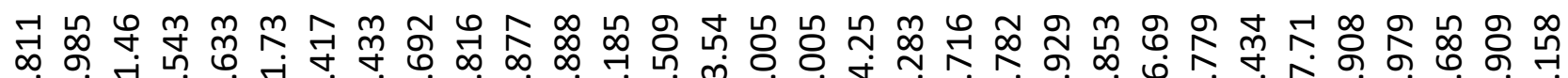

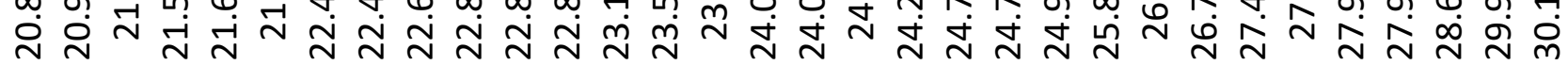

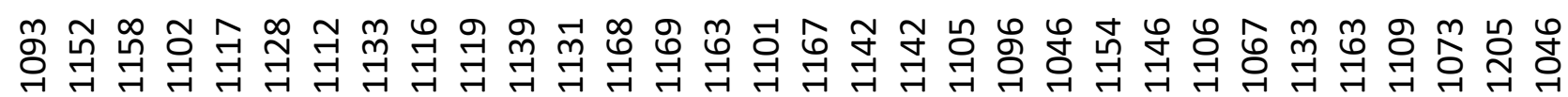

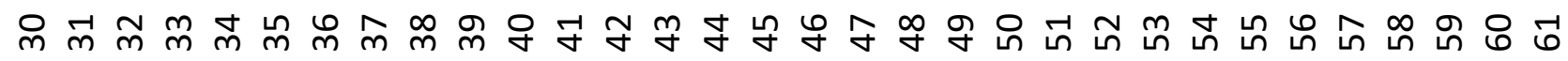

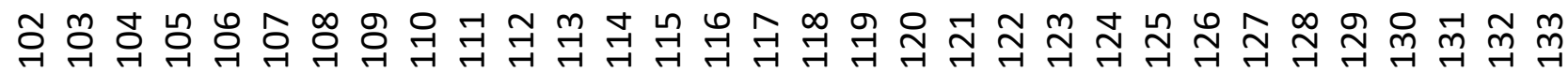

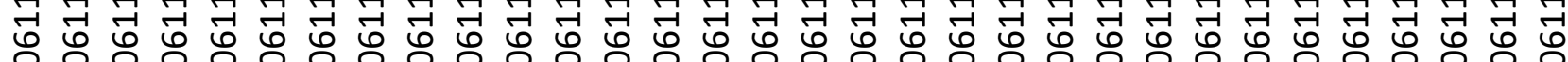

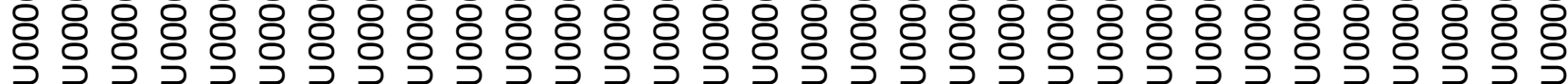

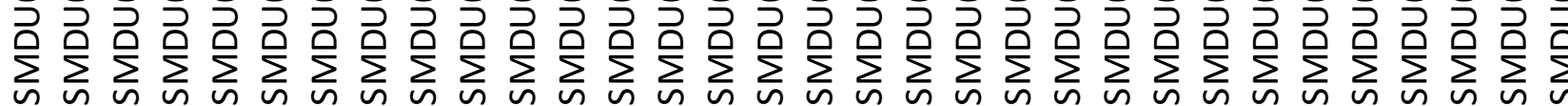

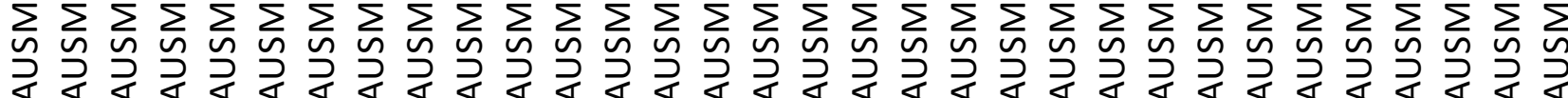




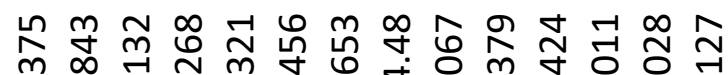
mं

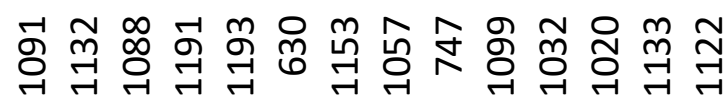

ㄴํำ

苟

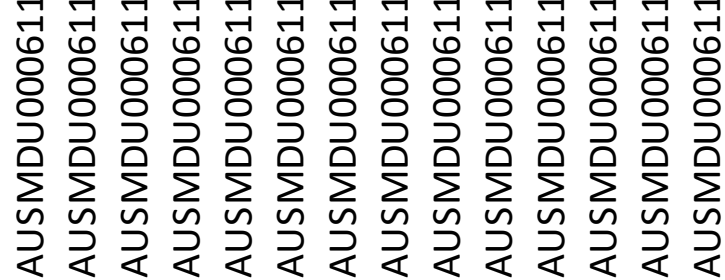

